\title{
An Analysis of the Line Art of Traditional Chinese Ceramic Sculpture
}

\author{
Linlin Shi \\ College of Technology and Art Jingdezhen Ceramic Institute
}

Keywords: Chinese tradition, Ceramic sculpture, Line art.

\begin{abstract}
This paper analyzes the essential attribute of "line" and the aesthetic concept of "line" of traditional Chinese ceramics, then analyzes the function of traditional ceramic sculpture and the application of "line" in the expression method, and finally analyzes the line art of traditional ceramic sculpture three functions in ceramic sculpture, including the expression of line in ceramic sculpture, the aesthetic value of line in ceramic sculpture and the line in ceramic sculpture. It is expected that the line art of Chinese traditional ceramic sculpture can be valued, studied, inherited and developed by relevant people.
\end{abstract}

\section{Introduction}

Chinese traditional ceramic art has developed since the Neolithic Age with a long history and culture. In the long history of Chinese ceramic culture, the aesthetics of ceramic sculptures are both unique and fascinating. The lines of Chinese traditional ceramic sculptures are vividly reflected in many handed down works. The artistic expression of line art in three-dimensional modeling is quite special and multifaceted. Generally, traditional Chinese ceramic sculptures mostly express the characteristics of decoration, painting, realism and freehand through "lines", and the culture of art works and the theme as well as beauty are passed to the audience. At the beginning, ceramics were produced only for use, and sculpture creations were used for the appearance or partial decoration of the shapes of people's everyday objects. With the advancement of human civilization, the audience has raised the artistic requirements for ceramic products. Ceramic sculpture products are no longer just the accessory decorations of daily necessities, but gradually become an art piece that satisfies the public aesthetics in an independent art form, and the use of line is also to enrich the changing art field continues to extend.

\section{The essential attribute of line and the aesthetic concept of line of traditional Chinese ceramics}

\section{(1) The essential attributes of "lines"}

In the visual representation of ceramic sculpture art, the artistic expression of lines and colors is different. In nature, color is objectively present and intuitively presented on the surface of objects, while figurative and pure lines do not exist. The essence of lines is either wide or narrow, thick or thin, long or short. The visual effect produced by the action. But in any matter, there is an aesthetic interpretation of the lines in people's vision, except that the size and shape of the lines are different, so the lines are the abstract visual effects of the form, the abstraction is its essential attribute, and it is also one of the powerful means in the work that can make the image become more abstract. In the creation process of traditional ceramic sculpture works, one of the most complex contents is to shape lines. The most difficult is to specifically integrate and aesthetically treat abstract and changeable lines. Compared with lines in painting works, the essence of lines in ceramic sculpture works is different from it. In ceramic sculpture, the definition of lines is under the light projection reflected in curved, straight, short or tall shadow works in the three-dimensional volume, or the line visual effect produced in the process of 360 degree continuous change of the contour of the sculpture in the circular sculpture works, as well as the high-level linear shape produced by the combination of the parts between the dignities. The expression of line beauty in ceramic sculptures is complex. It needs meticulous and exquisite ceramic carving technology to grasp the artistic essence of lines, which is also the form of artistic line expression that needs to be learned and 
inherited in modern ceramic sculpture creation.

(2) "Line" concept of traditional Chinese ceramics

Compared with the traditional sculptures of the western traditional Chinese sculptures, the oriental ethnic characteristics are distinct, and the "line" is an important element of the aesthetic "Orient" of Chinese art. The concept of "line" of traditional Chinese ceramics includes three aspects: the aesthetic expression of lines on the shape, the decorative expression of the texture of the material, the engraving and rendering of the painting and the content of the pattern, and the unique concept of the "line" by the creators of different eras. The pioneering development and application reflects the aesthetic value of line art. The "line" of traditional Chinese ceramic sculpture pays more attention to the movement, momentum, structure, form and charm of the object through lines or hidden or local, and freely organizes it to create a smooth and chic linear shape and space. Chinese ceramic sculpture is different from the straightforward expression of western expression, but pays more attention to the generalization of shaping the lines. For example, the "implication" and "freedom" methods make the works have their physical space, which is both subtle and dynamic but splendid. The artistic effect, which makes the creation of ceramic sculptures become more intrinsic and has an independent "life" atmosphere.

\section{The function of traditional ceramic sculpture and the use of lines in expression techniques}

\section{(1) The use of lines in the function of traditional ceramic sculpture}

The function of Chinese traditional ceramic sculpture is mainly used in tomb, small religious statues and the performance of utensils and building components. The terracotta warriors and horses in the mausoleum of the first emperor of Qin Dynasty are the most magnificent and complete ceramic tomb sculptures in China. The makers used highly realistic techniques to show a well-organized royal army vividly. The modeling performance of the terracotta warriors embodies the artistry of "lines": on the large body of the terracotta figures, the relatively dense short line strip body is used at the neckline, cuffs of the lapel to express the wrinkle texture of the clothing lines, and the dense line composition contained in the integrity is strengthened; the shoulder armor and breastplate are carved to show a series of square armor scales, forming regular and repeated lines, which are realistic and decorative. The head portrait created independently uses clear lines to deal with the facial features and subtle shallow relief to create a bright shadow effect, which embodies the painting characteristics of traditional Chinese sculpture. Details such as hair ornaments, whiskers, neck scarves, buttons, shoelaces, and the skin and texture of the fingernails of the hands are carefully traced in fine, delicate lines. The head, four hooves, saddle and other details of the terracotta horse are also carved with clear and short lines. The mane and tail of the horse are carved with hair-like lines to reflect the real texture. It can be said that the terracotta warriors and horses sculpture of Qin Shihuang tried to use the artistic expression of lines to interpret the representation and expression of the real world in the overall atmosphere of the form, thus reflecting the imitation and pursuit of real functions of tomb art.

(2) The application of lines in the expression techniques of traditional ceramic sculpture

There are great differences between Chinese and western traditional sculpture, especially the use of lines. The realism of Chinese traditional sculptures is basically reflected by the combination of lines or lines and floating sculptures. These lines are attached to the large body of the unified sculpture. Linear details Chinese traditional sculptors are more likely to grasp the expression of the works by observing the shape lines of objective objects. This has nothing to do with the influence of the aesthetic concept of Chinese calligraphy and painting art, while the traditional sculptures in the west mainly form the rich volume and light of the works through the relationship of the ups and downs, twists and connections of the mass effect. This stems from the fact that western traditional sculptors have applied scientific anatomical knowledge and spatial perspective to observe the objective world and carry out artistic creations in ancient Greece. Therefore, the traditional sculptures of the west are presented on the basis of a basic core framework. It is the sculptor's shape and expression of the structure and undulating mass of the body, which makes the work more objective and realistic. The uniqueness of the application of "lines" in traditional Chinese sculpture 
is strongly reflected in the modeling and expression of traditional ceramic sculpture. Because the material characteristics of ceramic materials to be fired at high temperature limit the expansion and richness of sculpture modeling in momentum and space creation design, so ceramic sculpture needs to maintain a relatively correct overall modeling to meet the requirements of firing, and the richness of the form is more dependent on the depiction and shaping of the line form. These factors jointly promote the use of "line" in traditional ceramic sculpture art.

\section{Line art of traditional Chinese ceramic sculpture}

\section{(1) The aesthetic expression of lines in traditional ceramic sculpture}

The creative content of traditional ceramic sculpture is rich in various styles, but also has a complex and exquisite production process. Among them, the representative Tri-colour Pottery of the Tang Dynasty highly praised by the art history and loved by the world, shows us the complexity and exquisite of the manufacturing process and its gorgeous artistic tension. The Tang Tri-colored Pottery are white, yellow, green, blue, brown and other multi-color glaze with three colors and two colors. Then they are made by two separate firing. After the first firing, the burnt figures and animals are colored again. The reserved naked burning parts without glaze, such as the cheeks, hands and feet of the figures, are dyed to reflect the skin's red color, including the hair crown, eyes, hair and eyebrows are painted with black color. This series of details are painted and filled by ceramic artists through skilled operation of lines. The Tang Tri-colored Pottery has full and abundant contour lines, mottled and incisive colorful lines, exquisite and vivid carving and painting lines on the surface of objects, and in the dynamic visual appreciation effect, the dynamic body space of the Tang Tri-colored Pottery is also composed of hundreds of thousands or even tens of thousands of small lines running through the surface of the works The reason why the Tang Tri-colored Pottery can become the art treasure in the development of traditional ceramic sculpture is that it has a unified grasp and pursuit of the artistic expression of works' ideas and materials, which contains the aesthetic expression of "lines" that can not be ignored.

\section{(2) The aesthetic value of lines in ceramic sculpture}

Line is the earliest and most intuitive means of understanding and expression in human aesthetic art activities. In the artistic expression of traditional ceramic sculpture works, line also has important aesthetic value. One of the most important forms of artistic works is the shaping of lines. Lines constitute the most basic and direct presentation of the body, so that the creator can present the image content of his creation to the audience through the lines. Lines can also show subtle and delicate changes in details, so that the creator can transmit rich and delicate emotions to the audience. Lines can show different artistic concepts through the form changes and arrangement and combination to make different texture effects. The creation of Chinese traditional ceramic sculpture works is influenced by the social background and the cultural atmosphere of the times, and the artistic application of lines is also different, which has high research value in aesthetics. No matter the ornamental ceramic sculpture, tomb type ceramic sculpture or religious ceramic sculpture, they all pay attention to the construction and sculpture of body and structure through different lines to present the works. Lines have a strong sense of organic life. They can be soft, rigid, straight, arbitrary, different in length and in all directions. They are all their artistic forms. Lines are the most widely used aesthetic element "tool" used by creators. In ceramic sculpture works, lines processing of form and space are used to present the aesthetic feeling and rhythm of life, so as to make the artistic form of ceramic sculpture more complete and perfect.

\section{(3) The role of lines in ceramic sculpture}

In the artistic expression of traditional ceramic sculpture, lines run through the whole process of design and production. Any ceramic sculpture work contains the visual carrier element of "line". The creation and design of ceramic sculpture work should first construct the frame of internal and external body through countless lines, then connect the point surface body and other elements in the space of the body, and then combine the decoration techniques to carve or depict the details on the surface of the whole body, Thus, vivid and rich ceramic sculptures can be created and show different artistic forms with different effects. 


\section{Conclusion}

Generally speaking, China's traditional ceramic sculptures mainly include tomb burial and religious belief. Tomb type ceramic sculptures, such as the well-known terracotta warriors and horses of the first emperor of the Qin Dynasty and the animal tomb figurines of figures in the tri-colored glazed pottery of the Tang Dynasty, can be seen as a means of visual art transmission. Religious beliefs, such as the Bodhisattva of Buddha and Arhat sculpture are different from the western ceramic sculptures of the same period in artistic aesthetics. The western ceramic sculpture attaches great importance to the expression of the mass of the objective form and volume, while the Chinese traditional ceramic sculpture attaches great importance to the interpretation of the artistic line form.

\section{Reference}

[1] Zeng Yi. 2019. Making Traditional Culture be Contemporary--on the Creation Experience of Ceramic Sculpture Works [J]. Ceramic Science and Art, 53 (08): $19+21$

[2] Ma Luyao. 2019. On the Application of Traditional Ceramic Art in Sculpture Teaching [J]. Art Education Research, (13): 142-143

[3] Lin Zhiming. 2019. Research on Traditional Ceramic Sculpture Techniques under the Concept of Modern Culture [J]. Ceramic Science and Art, 53 (07): 51

[4] Chen Zeci. 2019. On the Construction of Chinese Modern Ceramic Sculpture Form Based on Ontology Language [J]. Foshan Ceramics, 29 (02): 51-53

[5] Guo Yuwei, Zhao Ying, Zheng Enfeng. 2018. On the Role of Ceramic Sculpture in Environmental Art Space [J]. Ceramics, (12): 14-15

[6] Chen Danli. 2018. Artistic Language and Form Interpretation of Modern Ceramic Sculpture [J]. Jiangsu Ceramics, 51 (02): 14-15

[7] Chen Jiandu. 2018. Line Art of Traditional Chinese Ceramic Sculpture [J]. Jiangsu Ceramics, $51(02): 3-4$

[8] Yang Jianzhi. 2018. On the Combination of Traditional Ceramic Sculpture Techniques and Modern Cultural Concepts [J]. Jiangsu Ceramics, 51 (01): 70-71 